\title{
A True Aneurysm of the Profunda Femoris Artery: A Case Report and Review of the English Language Literature
}

Steven R. Posner, MD, Jonathan Wilensky, MD, Justin Dimick, MD, and Peter K. Henke, MD, Ann Arbor, Michigan

\begin{abstract}
A rare case of true aneurysm of the profunda femoris artery (PFA) is reported. Surgical management consisted of ligation of the aneurysm and decompression of the sac. Presentation, diagnosis, and treatment of true PFA aneurysms are discussed and the English language literature is comprehensively reviewed.
\end{abstract}

Aneurysms of the profunda femoris artery (PFA) are uncommon. Most result from injury to the vessel wall and have been reported as a consequence of penetrating injury, fracture, orthopedic procedures, or catheterization, and have even been induced by golf swing. ${ }^{1-4}$ True PFA aneurysms comprise $1-6.6 \%$ of all femoral artery aneurysms. $^{2,5-8}$ It has been postulated that aneurysmal change in this location is rare because of a protective muscular tunnel formed by the adductor magnus. ${ }^{6}$ Aneurysms may cause symptoms of distal venous congestion related to local venous compression, or neurologic symptoms from compression of the femoral or tibial nerve, be a source of distal emboli, thrombose causing ischemia, or rupture. ${ }^{5,6}$ We present a case of an atherosclerotic aneurysm of the PFA and review the English language literature.

\section{CASE REPORT}

The patient is an 80-year-old man with past medical history significant for hypertension and abdominal aortic aneurysm (AAA) repair 8 years earlier who presented with a 4-week history of left-sided foot drop, numbness, intermittent tingling, and progressive edema. He thought he had injured his foot while bowling. There was no

Section of Vascular Surgery, Department of Surgery, University of Michigan, School of Medicine, Ann Arbor, MI, USA.

Correspondence to: Peter K. Henke, MD, 2210D/0329 THCC, 1500 East Medical Center Drive, Ann Arbor, MI 48109-0329, USA, E-mail: henke@umich.edu

Ann Vasc Surg 2004; 18: 740-746

DOI: 10.1007/s10016-004-0116-4

(C) Annals of Vascular Surgery Inc.

Published online: 26 October 2004 previous history of claudication and prior ankle X-ray was negative. He had unintentionally 40 lost pounds over the preceding 10 months. The patient had a $100+$ pack-year smoking history. The patient's mother had an AAA.

On physical examination, blood pressure was 135/73. There was a large pulsatile mass in the left medial thigh. Bilateral femoral, popliteal, dorsalis pedis, and posterior tibial pulses were easily palpable. There was $2+$ edema of the left foot and leg to the knee. Sensation to light touch and pinprick was diminished to the knee. Thigh flexion and extension were normal, but the patient was unable to plantar or dorsiflex the left foot. The anklebrachial index was 1.1, with triphasic Doppler waveforms at the ankle. Lab evaluation was unremarkable.

Ultrasound of the left thigh showed a 5 to $6-\mathrm{cm}$ aneurysm of the PFA. Also noted were aneurysmal changes of the bilateral common iliac, common femoral, and popliteal arteries (Table I). Arteriography showed diffuse arteriomegaly, with bilateral common iliac aneurysms, and left-sided internal iliac, common femoral, and popliteal aneurysms. The PFA had aneurysmal dilatation with distal occlusion (Fig. 1). The superficial femoral artery (SFA) and outflow vessels to the foot were patent. Computed tomography of the thigh was also performed, confirming a 6-cm-diameter aneurysm of the PFA, extending $13 \mathrm{~cm}$ caudally (Fig. 2).

Chest radiography revealed a $6-\mathrm{cm}$ lung mass in the left lower lobe. Subsequent transbronchial biopsy was consistent with non-small-cell lung cancer. The decision was made to proceed with treatment of the PFA aneurysm prior to addressing the lung mass.

\section{Procedure}

After prepping the leg, a longitudinal incision was made directly over the aneurysm. The patient was found to have ectatic common and superficial femoral arteries. Dissection was continued down onto the PFA, reflecting 
Table I. Ultrasound measurements of pelvic and lower extremity arteries

\begin{tabular}{lllll}
\hline & $\begin{array}{l}\text { CIA } \\
(\mathrm{cm})\end{array}$ & $\begin{array}{l}\text { CFA } \\
(\mathrm{cm})\end{array}$ & $\begin{array}{l}\text { SFA } \\
(\mathrm{cm})\end{array}$ & $\begin{array}{l}\text { Popliteal artery } \\
(\mathrm{cm})\end{array}$ \\
\hline Right & 3.3 & 1.7 & 1 & 1.5 \\
Left & 3.5 & 1.6 & 1.3 & 1.3 \\
\hline
\end{tabular}

CFA, common femoral artery; CIA, common iliac artery; SFA, superficial femoral artery.

the femoral nerve, vastus medialis, and belly of the sartorius laterally. The femoral nerve ran along the lateral aspect of the aneurysm sac (Fig. 3). Proximal control was obtained just distal to the lateral circumflex femoral artery. Upon clamping the neck of the aneurysm, the aneurysm was no longer pulsatile and was opened. After removing a large amount of thrombus, there was minimal back-bleeding from a small outflow vessel, which was oversewn from within the aneurysm sac. The SFA was widely patent, making vascular reconstruction unnecessary. The neck of the aneurysm was therefore ligated, and the leg closed in layers.

\section{Outcome}

On follow-up the patient had palpable foot pulses. There was a persistent left foot drop, but his lower extremity edema had mostly resolved. His lung cancer was found to have crossed the major fissure, was deemed nonresectable, and was therefore treated with chemotherapy and radiation therapy. He was alive 12 months after the procedure.

\section{DISCUSSION}

We reviewed 46 patients with PFA aneurysms reported in the English language literature (Table II). Data with sufficient detail for analysis (some incomplete) were available for 42 patients. True aneurysms of the PFA are much more common in men $(92 \%)$ than in women. This pattern of incidence is comparable to the male predominance of all femoral aneurysms $(89-100 \% \text {. })^{5-8,11,43}$. Average age at presentation of patients with PFA aneurysms is $73.5 \pm 10$ years. These are bilateral in only $5 \%$ of patients; this is in contrast to all femoral aneurysms, which are bilateral in the majority. ${ }^{5-7,11,43}$

Patients with PFA aneurysms often have aneurysms elsewhere: of 31 patients with available data, 20 (65\%) had at least one other aneurysm. Twelve $(39 \%)$ had an (AAA) and $15(38 \%)$ had three or more aneurysms. These findings are consistent with most studies of patients with femoral aneurysms, which have reported multiple aneurysms in 58-69\% of patients and AAA in $28-58 \%$ of patients ${ }^{5-7,43}$

Presenting symptom history was available for 34 patients. The most common presentation was groin or proximal thigh swelling and pain, occurring in $17(50 \%)$ patients. Eight (24\%) patients presented with only thigh

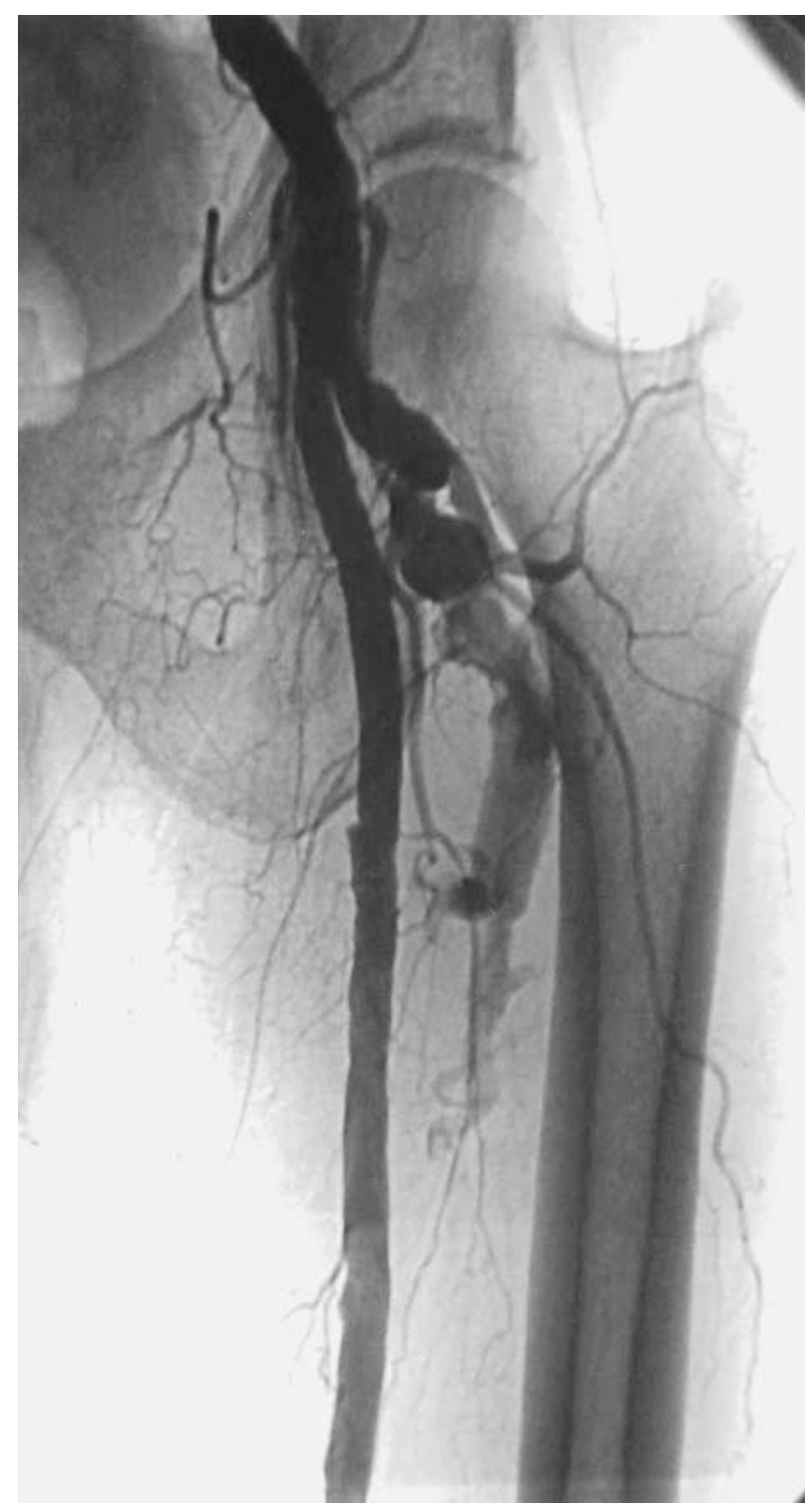

Fig. 1. Arteriogram shows aneurysmal change and occlusion of the PFA.

swelling or an asymptomatic pulsatile mass. Three patients had evidence of distal emboli, two of whom had no other aneurysms and four who had digital ischemia. It is difficult to account for distal embolization from an isolated PFA aneurysm. One proposed explanation is retrograde passage of clot into the SFA and subsequent embolization, due to compression or manipulation of the aneurysm. ${ }^{21}$ Rupture is a common presentation for PFA aneurysms, and occurred in 18 patients (44\%). All ruptures presented with both pain and swelling of the groin or thigh. Foot drop is an uncommon presentation, occurring in only one or two other patients. In our patient, the foot drop was likely related to compression of the tibial nerve by his popliteal aneurysm and was therefore not improved after resection of the aneurysm sac. 


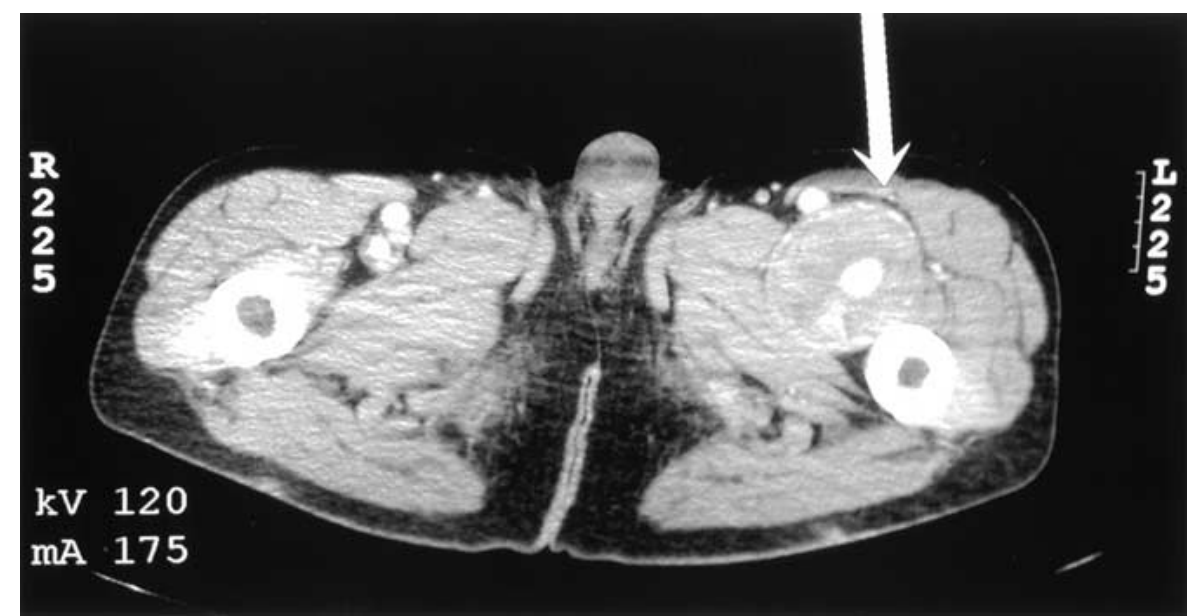

Fig. 2. Computed tomography of proximal thigh: arrow indicates 6-cm aneurysm of the PFA.

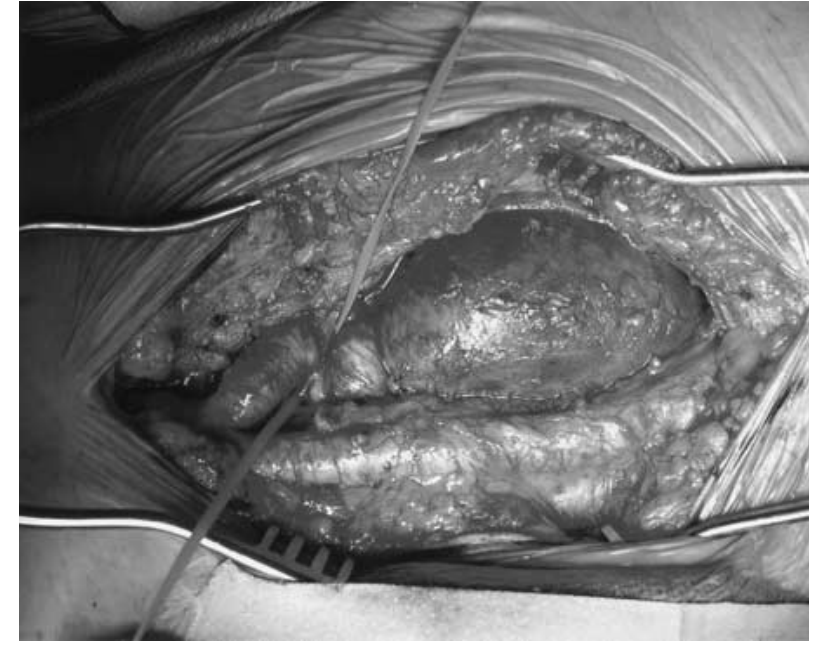

Fig. 3. Left proximal thigh with large PFA aneurysm at exploration. The patient's head is to the left.

The size of PFA aneurysms is not well documented in the literature. In one series the average size was $3.3 \pm$ $1.8 \mathrm{~cm} .{ }^{43}$ PFA aneurysms tend to present at a larger size than that of femoral aneurysms. Average size was $7.4 \pm$ $3 \mathrm{~cm}$ in 28 patients from this review. Aneurysms that were ruptured on presentation tended to be larger than those that were not $(8.8 \pm 3.0 \mathrm{~cm}$ vs. $6.3 \pm 3.1 \mathrm{~cm}, p=$ $0.04)$. The larger size at presentation compared with that of all femoral aneurysms is likely due to the deeper position of the PFA in the thigh, which may delay diagnosis, and may also explain the increased incidence of rupture: 18 of $41(44 \%)$ patients presented with rupture, compared with $2-24 \%$ of patients with femoral artery aneurysms $5,6,43,44$

Because of the larger size at diagnosis and the greater incidence of rupture, all PFA aneurysms should be treated, with or without reconstruction, on diagnosis. Surgical treatment consisted of ligation in 25 patients, reconstruction in 16 patients, and aneurysmorrhaphy in 1 patient. Prosthetic graft material was used for recon- struction in 11 patients (6 with Dacron, ${ }^{5,10,21,24,26,32} 4$ with ePTFE, ${ }^{18,20,34,37}$ and 1 human umbilical vein ${ }^{17}$ ) and greater saphenous vein graft in 4 patients. ${ }^{12,31,42}$ The form of repair could not be ascertained for two patients. $^{38,41}$

The decision to ligate or revascularize a PFA aneurysm depends on the patency of the femoral-popliteal outflow and the feasibility of repair. Most reports recommend reconstruction of the aneurysm with an interposition graft to preserve PFA flow, especially in the setting of distal femoral-popliteal outflow tract occlusion. ${ }^{12,15,24}$ However, there was no significant difference in outcome in the 11 patients reviewed with femoral-popliteal outflow tract occlusion (based on the absence of pedal pulses, when angiographic data were not available) according to the form of operative therapy: only four progressed to amputation, one of three were treated with ligation, two of seven underwent reconstruction, and one patient underwent aneurysmorrhaphy. No patient with intact distal pulses required amputation.

Patients with ruptured aneurysms usually have enough anatomic distortion to make reconstruction difficult at best. Of 18 patients who had a ruptured PFA aneurysm, 14 were treated by ligation, with only one amputation. Distal pulses were present in 10 of these patients, absent in 2, and unknown in 6. Therefore, it appears that patients with rupture with intact distal pulses may safely undergo ligation without revascularization. However, in the setting of rupture with absent distal pulses, there are not enough data to document the safety of ligation. In these cases, efforts should be made to perform revascularization, if feasible.

Even though some patients, according to our review of the literature, may not require reconstruction, we recommend reconstruction in all patients with occlusion of the distal outflow. This may be done with autogenous vein graft or prosthetic material. Aneurysmectomy with ligation of the inflow and outflow may be performed safely if there is adequate femoral-popliteal outflow and limited atherosclerotic occlusive disease. Published concerns that operative repair in the setting of SFA occlusion has a poor outcome with a high rate of subsequent amputation ${ }^{5}$ have 


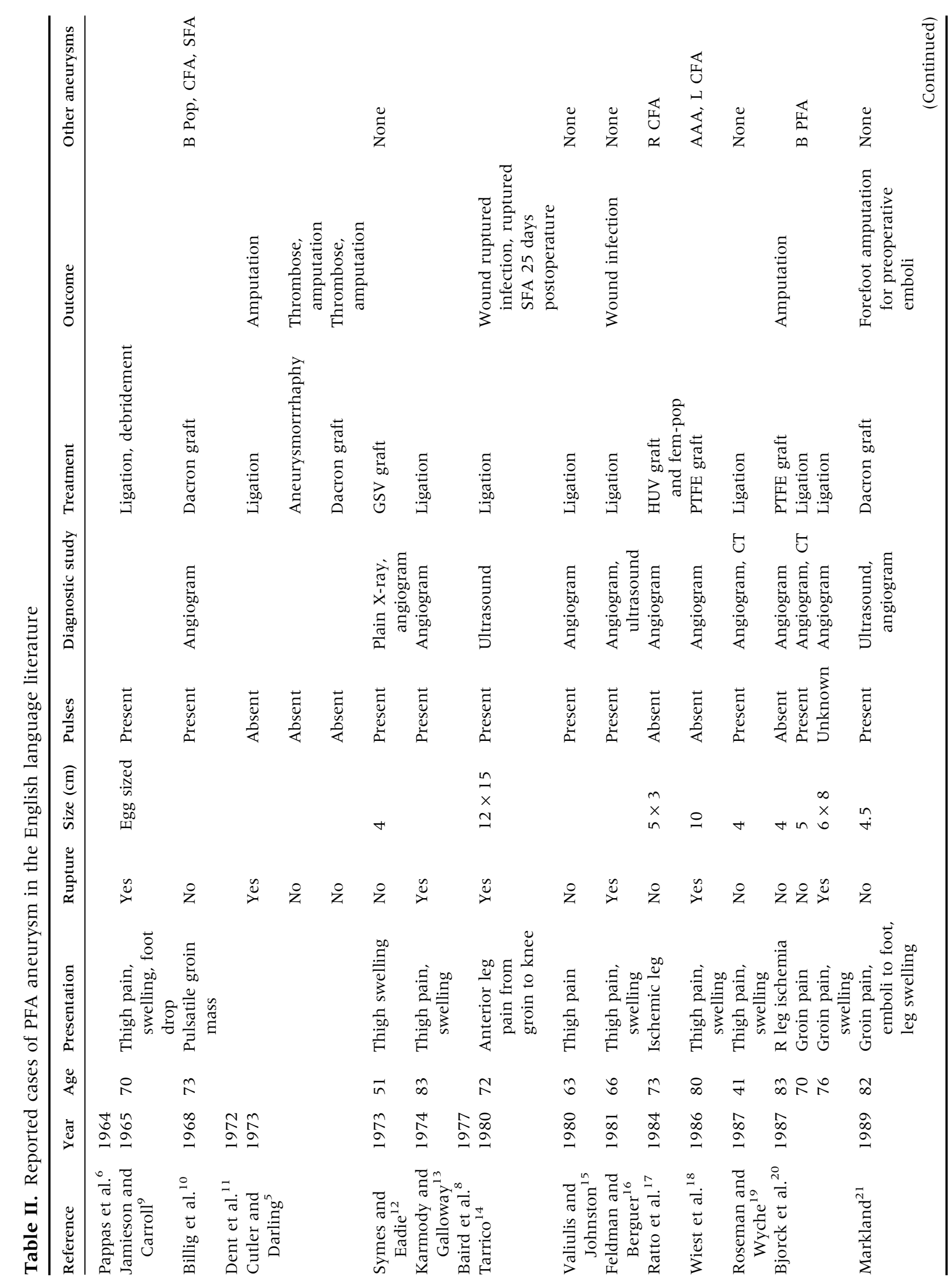




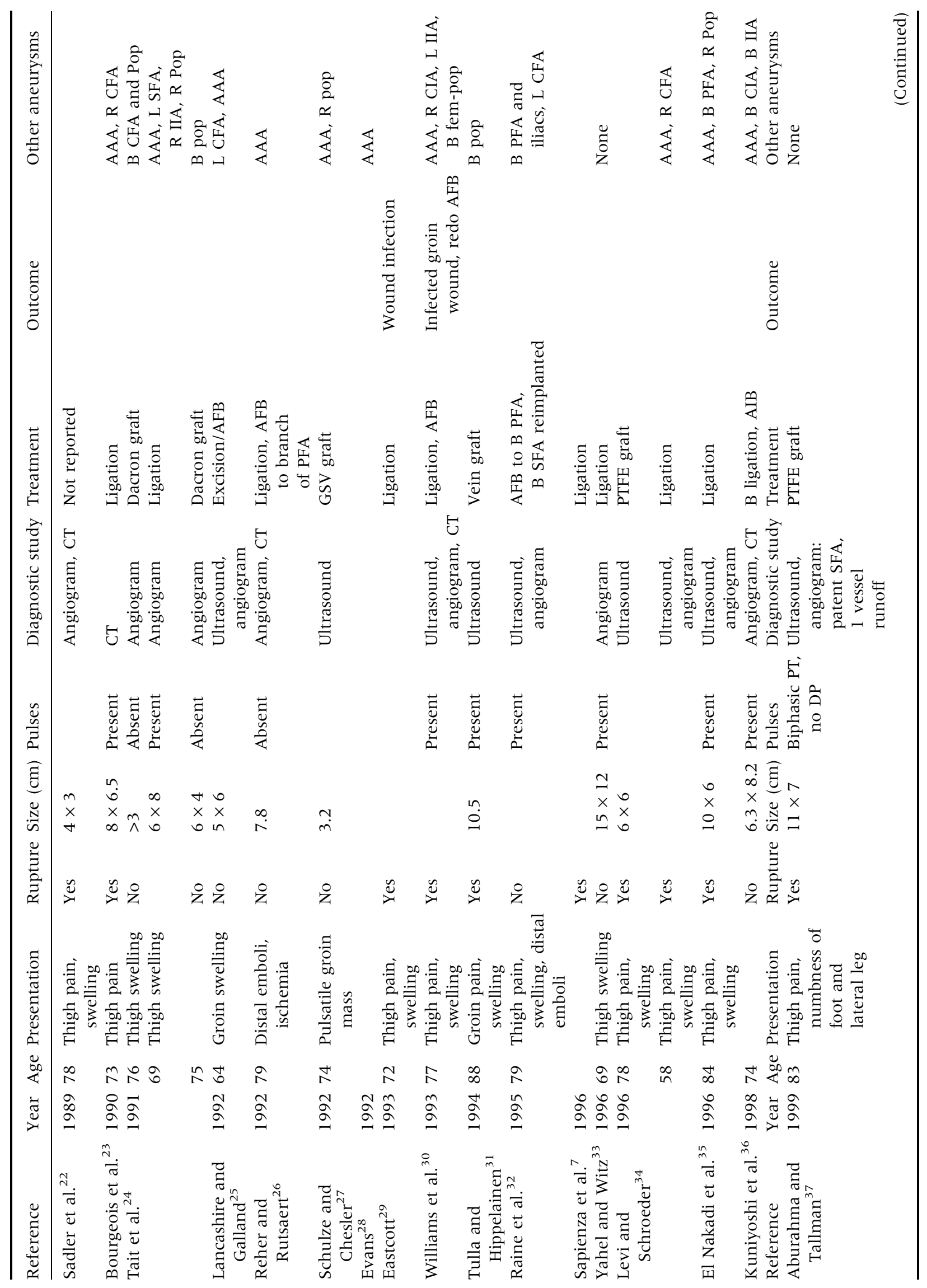




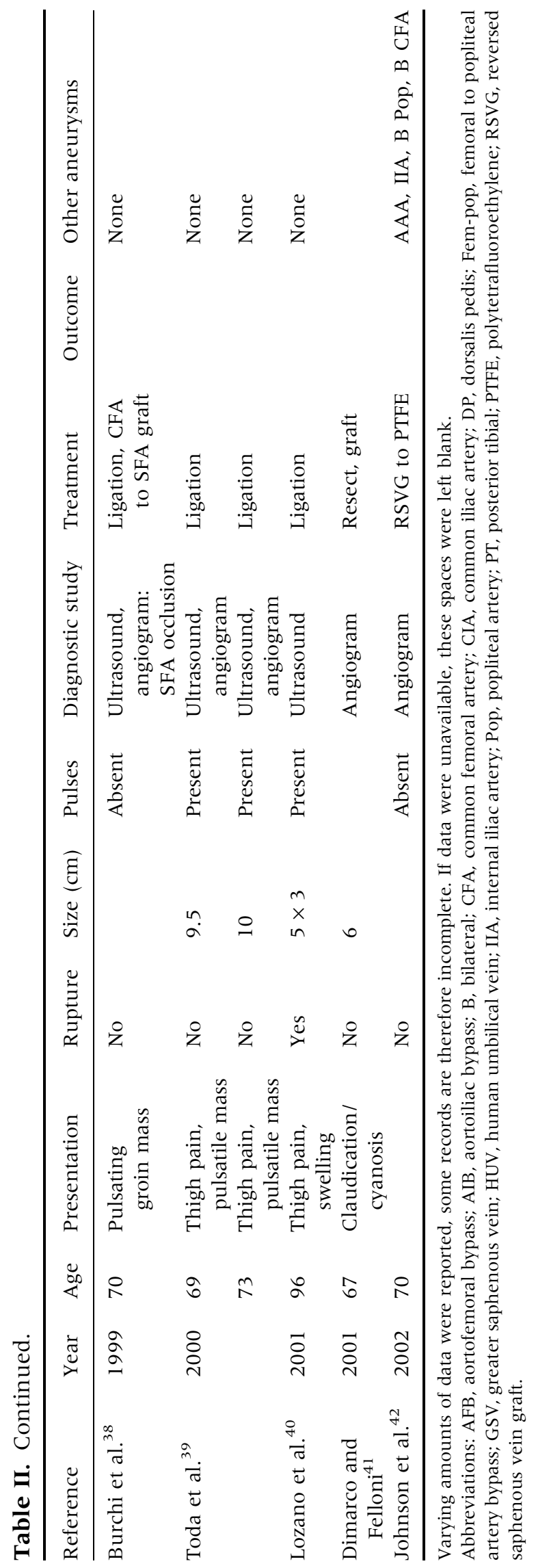

not been borne out by careful review of the literature. There are no convincing data to support reconstruction over ligation in the setting of ruptured PFA aneurysm - this decision should be based on adequacy of distal perfusion as assessed clinically at the time of operation.

In summary, an aneurysm of the PFA is a rare problem, comprising only $1-6.6 \%$ of all femoral aneurysms. The pathophysiology of patients with aneurysmosis such as this probably have an as-yet undefined connective tissue metabolism defect. Molecular studies comparing patients with isolated AAA and those with multiple aneurysms have not yet been done on a large scale. PFA aneurysms tend to present at a larger size and have a higher incidence of rupture than that of femoral aneurysms. Revascularization in the setting of a patent femoral-popliteal outflow tract may not be necessary, but should be pursued if feasible. When the distal femoral-popliteal outflow tract is occluded, reconstruction is strongly recommended, whereas simple ligation may be safely performed in the setting of adequate outflow to the foot.

\section{REFERENCES}

1. Calligaro K, Savarese R, Goldberg D, Doerr K, Dougherty M, DeLaurentis D. Deep femoral pseudoaneurysm caused by acute trunk and hip torsion. Cardiovasc. Surg. 1993;1:392394.

2. Berry M, Van Schil P, Vanmaele R, De Vries D. Infected false aneurysm after puncture of an aneurysm of the deep femoral artery. Eur. J. Vasc. Surg. 1994;8:372-374.

3. Loubeau J, Bahnson H. Traumatic false aneurysm and arteriovenous fistula of the profunda femoris artery: surgical management and review of the literature. Surgery 1977;81:222-227.

4. Murphy P, Geoghegan J, Austin O, More-O'Ferrall R, Quinlan W, Keavneny T. Pseudoaneurysm of the profunda femoris artery due to intertrochanteric fracture of the hip. Arch. Othop. Trauma Surg. 1996;119:117-118.

5. Cutler B, Darling R. Surgical management of arteriosclerotic femoral aneurysms. Surgery 1973;74:764-773.

6. Pappas G, James J, Bernatz P, Schriger A. Femoral aneurysms. J.A.M.A. 1964;190:489-493.

7. Sapienza P, Mingoli A, Feldhaus R, di Marzo L, Cavallari N, Cavallaro A. Femoral artery aneurysms: long-term followup and results of surgical treatment. Cardiovasc. Surg. 1996; 4:181-184.

8. Baird R, Gurry R, Kellam J, Plume S. Arteriosclerotic femoral artery aneurysms. Can. Med. Assoc. J. 1977;1 17:1306-1307.

9. Jamieson W, Carroll S. Ruptured profunda femoral aneurysm presenting as thrombophlebitis. Med. Ser. J. Can. 1965;21:193-198.

10. Billig D, Jordan P, DeBakey M. Arteriosclerotic aneurysm of the profunda femoris artery: report of a case with successful surgical repair. Cardiovasc. Res. Cent. Bull. 1968;6:139-141.

11. Dent T, Lindenenauer S, Ernst C, Fry W. Multiple arteriosclerotic arterial aneurysms. Arch. Surg. 1972;105:338-344.

12. Symes J, Eadie D. Solitary arteriosclerotic aneurysm of the profunda femoris artery. J. Cardiovasc. Surg. 1973;14:220222.

13. Karmody A, Galloway J. Aneurysm of the profunda femoris artery. J. R. Coll. Surg. Edinb. 1972;17:261-263.

14. Tarrico A. Ruptured aneurysm of profunda femoris (sic). N.Y. State J. Med. 1980;80:960-962. 
15. Valiulis A, Johnston K. Isolated arteriosclerotic aneurysm of the profunda femoris artery. J. Cardiovasc. Surg. 1980;21:498-500.

16. Feldman A, Berguer R. Rupture of isolated atherosclerotic aneurysm of the lateral femoral circumflex artery. Surgery 1981;90:914-916.

17. Ratto G, Sacco A, Canepa G, Motta G. Atherosclerotic aneurysm of the deep femoral artery. J. Cardiovasc. Surg. 1984;25:574-576.

18. Wiest J, Mukherjee D, Inahara T. Ruptured aneurysm of the profunda femoris artery. J. Vasc. Surg. 1986;4:406-409.

19. Roseman J, Wyche D. True aneurysm of the profunda femoris artery. J. Cardiovasc. Surg. 1987;28:701-705.

20. Bjorck C, Bergqvist D, Ljungner H, Rimer U. Atherosclerotic aneurysm of the deep femoral artery. Acta Chir. Scand. 1987;153:137-142.

21. Markland C. Primary atherosclerotic aneurysm of the profunda femoris artery associated with distal embolization. Ann. Vasc. Surg. 1989;3:389-391.

22. Sadler L, Bolden R, Lenkey J. Diagnosis of a ruptured deep femoral artery aneurysm-a case report. Angiology 1989;40:..

23. Bourgeois D, Nolan R, Brown P. Computed tomography of ruptured aneurysm of the profunda femoris artery. Clin. Imaging 1990; 14:228-230.

24. Tait W, Vohra R, Carr H, Thomson G, Walker M. True profunda femoris aneurysms: are they more dangerous than other atherosclerotic aneurysms of the femoropopliteal segment?. Ann. Vasc. Surg. 1991;5:92-95.

25. Lancashire M, Galland R. Aneurysm of the lateral circumflex femoral artery in association with multiple atherosclerotic aneurysms. Ann. Vasc. Surg. 1992;6:289-291.

26. Reher S, Rutsaert R. Atherosclerotic aneurysm of the deep femoral artery [letter]. Eur. J. Vasc. Surg. 1992;6:226.

27. Schulze K, Chester J. Aneurysm of the profunda femoris artery. Aust. N. Z. J. Surg. 1994;64:69-70.

28. Evans H. Atherosclerotic aneurysm of the deep femoral artery [letter]. Eur. J. Vasc. Surg. 1992;6:450.

29. Eastcott HH. Arterial Surgery. London: Pittman, 1993, p 441.
30. Williams I, Baildam A, Eyers P, McCollum C. Rupture of a true profunda femoris aneurysm. J. Cardiovasc. Surg. 1993;37:17-20.

31. Tulla H, Hippelainen M. Ruptured massive aneurysm of the deep femoral artery. Ann. Chir. Gynaecol. 1994;83:76-78.

32. Raine N, Magee T, Galland R. Thigh embolization in association with bilateral profunda femoris aneurysms. Eur. J. Vasc. Endovasc. Surg. 1995;9:491-492.

33. Yahel J, Witz M. Isolated true atherosclerotic aneurysms of the deep femoral artery. J. Cardiovasc. Surg. 1996;37:17-20.

34. Levi N, Schroeder T. Rupture of true profunda femoris artery aneurysms. J. Cardiovasc. Surg. 1996;37:117-118.

35. El Nakadi B, Bertrand S, Farran M. Deep femoral artery aneurysm rupture. J. Cardiovasc. Surg. 1996;37:353-354.

36. Kuniyoshi Y, Koja K, Akasaki M, et al. Successful treatment of bilateral deep femoral aneurysms and multiple iliac aneurysms associated with severe aortic valve disease. Surg. Today 1998;28:1206-1209.

37. Aburahma A, Tallman T. Ruptured isolated true atherosclerotic aneurysm of the deep femoral artery. J. Cardiovasc. Surg. 1999;40:45-47.

38. Burchi C, Cavallaro G, Amato D, Cavallaro A. Isolated true atherosclerotic anerysm of the profunda femoris artery. J. Cardiovasc. Surg. 1999;40:577-581.

39. Toda R, Uda T, Watanabe S, Hisashi Y, Moriyama YA. Surgical repair of a solitary deep femoral arterial aneurysm: report of two cases. Surg. Today 2000;30:481-483.

40. Lozano F, Sanchez-Fernandez J, Gomez A. Ruptured aneurysm of the deep femoral artery. Case report and historical review. J. Cardiovasc. Surg. 2001;42:821-824.

41. Dimarco L, Felloni M. Aneurysm of the profunda femoris artery [letter]. J. Cardiovasc. Surg. 2001;42:437.

42. Johnson C, Goff J, Rehrig S, Hadro N. Asymptomatic profunda femoris artery aneurysm: diagnosis and rationale for management. Eur. J. Vasc. Endovasc. Surg. 2002;24:91-92.

43. Graham L, Zelenock G, Whitehouse W, Erlandson E, Dent T, Lindenauer S. Clinical significance of arteriosclerotic femoral artery aneurysms. Arch. Surg. 1980;115:502-507.

44. Levi N, Schroeder T. Arteriosclerotic femoral artery aneurysms. J. Cardiovasc. Surg. 1997;38:335-338. 